MATHEMATICAL MODELLING OF

POPULATION DYNAMICS

BANACH CENTER PUBLICATIONS, VOLUME 63

INSTITUTE OF MATHEMATICS

POLISH ACADEMY OF SCIENCES

WARSZAWA 2004

\title{
TIME DELAYS IN PROLIFERATION AND APOPTOSIS FOR SOLID AVASCULAR TUMOUR
}

\author{
URSZULA FORYŚ \\ Institute of Applied Mathematics and Mechanics \\ Faculty of Mathematics, Informatics 85 Mechanics \\ Warsaw University, Banacha 2, 02-097 Warszawa, Poland \\ E-mail: urszula@mimuw.edu.pl \\ MIKHAIL KOLEV \\ Institute of Applied Mathematics and Mechanics \\ Warsaw University, Banacha 2, 02-097 Warszawa, Poland \\ and \\ South-West University, Blagoevgrad, Bulgaria \\ E-mail: mkkolev@yahoo.com
}

\begin{abstract}
The role of time delays in solid avascular tumour growth is considered. The model is formulated in terms of a reaction-diffusion equation and mass conservation law. Two main processes are taken into account-proliferation and apoptosis. We introduce time delay first in underlying apoptosis only and then in both processes. In the absence of necrosis the model reduces to one ordinary differential equation with one discrete delay which describes the changes of tumour radius.

Basic properties of the model depending on the magnitude of delay are studied. Nonnegativity of solutions is investigated. Steady state and the Hopf bifurcation analysis are presented. The results are illustrated by computer simulations.
\end{abstract}

1. Introduction. The aim of this paper is to present complementary analysis of the model considered and studied in $[3,4,5]$. The model describes the evolution of avascular multicellular spheroid (MCS) which may be also interpreted as the first stage of solid avascular tumour's growth. The basic idea of such models comes from [10]. Following

2000 Mathematics Subject Classification: 35K57, 62P10, 93A30.

Key words and phrases: solid avascular tumour, reaction-diffusion equation, delay-differential equation, stability, Hopf bifurcation.

This paper was prepared in the framework of RTN "Using Mathematical Modelling and Computer Simulation to Improve Cancer Therapy", grant HPRN-CT 2000-00105.

The paper is in final form and no version of it will be published elsewhere. 
the ideas presented in [5], analysis of the models with delay in proliferation process and regulatory apoptosis was done in $[3,4]$ in the framework of delay differential equations. In this paper (as in $[3,4]$ ) we focus on the case of uniformly proliferating tumour, i.e. MCS without a necrotic core inside. We introduce time delay in underlying apoptosis. This delay was not considered in $[3,4,5]$, but there are some suggestions that it may play similar role as in the case of proliferation process. In [5] delay was connected with the process of regulatory apoptosis which is caused by the presence of different inhibitory or growth factors. It is known that the process of underlying apoptosis is also delayed comparing to the trigger of this process and the modern studies (see e.g. [13]) show that this delay may be large.

Next we introduce time delay into both processes, i.e. proliferation and apoptosis. We study the case when these delays are equal. It is the first approximation to the analysis of the model with two different delays. This last case may be more interesting but also more difficult from the analytical point of view and therefore, we start our analysis from the simplest case.

At the end of this paper we compare dynamics of the models studied in $[3,4]$ and in this paper. We focus on the possibility of occurrence of oscillations which is one of the most important consequences of delays pointed out in [5]. We would like to choose the best way of introducing delays in the model of MCS growth.

At the beginning, we formulate the basic model (for details concerning this one and many similar models see $[1,3,4,5,6,7,10]$ ). To simplify the model equations, we assume that the growth of MCS is symmetric. We denote the space coordinate by $r$ (the radius of MCS). We focus on the changes of the outer MCS (tumour) radius $R(t)$ in time $t$. These changes depend on the nutrient concentration $\sigma(r, t)$ at the radius $r$ from the center of MCS and time $t$. The changes of $\sigma$ are described by the simple reaction-diffusion equation of the form

$$
\frac{1}{r^{2}} \frac{\partial}{\partial r}\left(r^{2} \frac{\partial \sigma}{\partial r}\right)=a,
$$

where the left-hand side of Eq. (1) represents the Laplacian in spherical coordinates and $a$ denotes the constant rate of nutrient consumption by tumour cells.

The changes of MCS volume are governed by the mass conservation law

$$
\frac{1}{4 \pi} \frac{d}{d t}\left(\frac{4}{3} \pi R^{3}\right)=S-Q
$$

where $S$ and $Q$ denote the net rates of proliferation and apoptosis, respectively. In the case without delay we assume that

$$
S(t)=\int_{0}^{R(t)} s \sigma(r, t) r^{2} d r, \quad Q(t)=\int_{0}^{R(t)} s c r^{2} d r
$$

where $s, c>0$ are some constants. For simplicity, in the next sections we assume that $s=1$.

Eqs. (1), (2) and (3) are considered with the following boundary and initial conditions:

$$
\sigma(R(t), t)=\sigma_{e}, \quad \frac{\partial \sigma}{\partial r}(0, t)=0, \quad R(0)=R_{0}
$$


where $\sigma_{e}$ denotes the constant external nutrient concentration and it is reasonable to assume that

$$
\sigma_{e}>c
$$

Calculating $\sigma$ from Eq. (1) with conditions defined by Eq. (4) we obtain the formula

$$
\sigma(r, t)=\sigma_{e}-\frac{a}{6}\left(R^{2}(t)-r^{2}\right)
$$

which combines the changes of our variables - the outer tumour radius and the nutrient concentration. Let us note that the condition for positivity of $\sigma(r, t)$ will be guaranteed if the outer MCS radius $R(t)$ is sufficiently small, namely if

$$
R(t) \leq R_{c}=\left(\frac{6 \sigma_{e}}{a}\right)^{\frac{1}{2}}
$$

The biological implication of this fact is that the model is not valid for $R(t)>R_{c}$, because then the process of formation of necrotic core inside the MCS appears.

2. Presentation of the model with delay in underlying apoptosis. In this section we study the model with delay in underlying apoptosis. Following the ideas presented in [5] for proliferation, we assume that there is a delay $(\tau>0$, constant for simplicity) between the time at which a signal for apoptosis is sent and the time at which apoptosis occurs. Therefore, instead of the formula presented in Eq. (3) we consider the following:

$$
Q(t)=\int_{0}^{R(t-\tau)} c r^{2} d r
$$

On the basis of Eq. (8) we obtain

$$
3 R^{2} \frac{d R}{d t}(t)=\sigma_{e} R^{3}(t)-\frac{a R^{5}(t)}{15}-c R^{3}(t-\tau)
$$

Let denote $x(t)=R^{3}(t)$. Using Eq. (9) we formulate the model

$$
\dot{x}(t)=-c x(t-\tau)+\sigma_{e} x(t)-\frac{a}{15} x^{\frac{5}{3}}(t),
$$

which we study with a nonnegative initial function $x^{0}(t)$, for $t \in[-\tau, 0]$. It is obvious that for every continuous $x^{0}$, there exits unique solution to Eq. (10), but it is not necessarily nonnegative for nonnegative initial $x^{0}$. Consider the initial function

$$
x^{0}(t)=-\alpha t, \quad \alpha>0, \quad t \in[-\tau, 0] .
$$

For the above function we obtain the solution to Eq. (10) with the property

$$
\dot{x}(0)=-c \alpha \tau<0, \quad x(0)=0,
$$

which means that the solution becomes negative. Moreover, there are no clear conditions guaranteeing nonnegativity of solutions in this case.

If we assume that the solution is nonnegative, then it is obviously defined for every $t>0$, because the inequality $\dot{x} \leq \sigma_{e} x$ is satisfied in such a case. On the other hand, if the solution becomes negative, then it is also bounded and therefore, it is defined for every $t>0$. Hence, we can study the asymptotic behaviour of solutions to Eq. (10). 
There are two stationary solutions to Eq. (10) - the trivial one and the positive nontrivial $\bar{x}=\left(\frac{15\left(\sigma_{e}-c\right)}{a}\right)^{\frac{3}{2}}$.

LEMma 1. The trivial stationary solution to Eq. (10) is unstable for every $\tau \geq 0$. The nontrivial solution $\bar{x}$ is stable independently of the delay for $4 c<\sigma_{e}$. If $\left.4 c\right\rangle \sigma_{e}$, then there exists a threshold value $\tau_{c}$ of the delay such that the solution is stable for $\tau<\tau_{c}$ and unstable otherwise. The Hopf bifurcation occurs at $\tau_{c}$.

Proof. Linearizing (see e.g. [11]) Eq. (10) around the trivial solution one obtains

$$
\dot{x}(t)=\sigma_{e} x(t)-c x(t-\tau) .
$$

Ineq. (5) implies that this solution is unstable for $\tau=0$ and therefore, it is unstable for every positive delay (for details see $[8,9]$ ).

For the second stationary solution we have the linearized equation of the form

$$
\dot{x}(t)=A x(t)+B x(t-\tau)
$$

where $A=\frac{5 c-2 \sigma_{e}}{3}$ and $B=-c$. We see that $A+B<0$ and $A-B=\frac{8 c-2 \sigma_{e}}{3}$. Corollary 5 in [8] implies that if $4 c<\sigma_{e}$, then the solution is stable independently of the delay, but if $4 c>\sigma_{e}$, then there exists the threshold value $\tau_{c}=\frac{1}{\sqrt{B^{2}-A^{2}}} \arccos \left(-\frac{A}{B}\right)$ such that the Hopf bifurcation occurs at this point.

3. Presentation of the model with delays in both processes. In this section we consider the case when both processes, underlying apoptosis and proliferation, are delayed. In this case Eq. (3) changes to

$$
S(t)=\int_{0}^{R\left(t-\tau_{1}\right)} s \sigma\left(r, t-\tau_{1}\right) r^{2} d r, \quad Q(t)=\int_{0}^{R\left(t-\tau_{2}\right)} c r^{2} d r
$$

From the biological point of view, both delays play similar role in cellular processes and therefore, their magnitudes are similar in most cases. Hence, we can consider the case $\tau_{1}=\tau_{2}$. The case $\tau_{1} \neq \tau_{2}$ is also interesting but more complicated from the analytical point of view.

In this paper we study the case $\tau_{1}=\tau_{2}=\tau$, which is the simpler one. Then our equation takes the form

$$
\dot{x}(t)=\left(\sigma_{e}-c\right) x(t-\tau)-\frac{a}{15} x^{\frac{5}{3}}(t-\tau) .
$$

It is easy to see that for every initial continuous function $x^{0}(h), h \in[-\tau, 0]$ there exists unique solution to Eq. (13), because on every time interval of the form $[n \tau,(n+1) \tau], n \in$ $\mathbf{N}$ the solution can be calculated as

$$
x(t)=x(n \tau)+\int_{n \tau}^{t}\left(\left(\sigma_{e}-c\right) x(s-\tau)-\frac{a}{15} x^{\frac{5}{3}}(s-\tau)\right) d s .
$$

This formula is called the step method (see e.g. [11]). As in the case studied in the previous section we define a nonnegative initial function $x^{0}(t)$ for $t \in[-\tau, 0]$ and consider nonnegativity of solutions to Eq. (13). Defining the auxiliary function $f(z)=\left(\sigma_{e}-c\right) z-$ $\frac{a}{15} z^{\frac{5}{3}}$ we see that $f(z) \geq 0$ for $z \in\left[0,\left(15 \frac{\sigma_{e}-c}{a}\right)^{\frac{3}{2}}\right]$ and $f(z)<0$ for other values of $z>0$. It is easy to check that if $x^{0}(h)=-\frac{\alpha h}{\tau}$, then $\dot{x}(0)=f\left(x^{0}(-\tau)\right)=f(\alpha)<0$ 
for $\alpha>\left(15 \frac{\sigma_{e}-c}{a}\right)^{\frac{3}{2}}$. Therefore, the solution to Eq. (13) may be negative for nonnegative initial function.

Assume now that $x^{0}(h) \in[0, \bar{x}]$. It is easy to see that maximal value of $f$ is equal to $f_{m}=\frac{54}{5} \frac{\left(\sigma_{e}-c\right)^{\frac{5}{2}}}{a^{\frac{3}{2}}}$ for $\tilde{z}=\left(\frac{9}{a}\left(\sigma_{e}-c\right)\right)^{\frac{3}{2}}$. Following the ideas presented in [2] we conclude that for $t \in[0, \tau]$,

$$
x(t) \leq \bar{x}+\int_{-\tau}^{t-\tau} f_{m} d s \leq \bar{x}+f_{m} \tau=x_{m} .
$$

Now, either $x(t) \in[0, \bar{x}]$ for every $t \geq 0$ or there exists $t>0$ such that $x(t)>\bar{x}$. If $x(t)>\bar{x}$, then there exists a first point $\bar{t}>0$ such that $x(\bar{t})=\bar{x}$ and $\dot{x}(\bar{t}) \geq 0$. But for every $t \in[\bar{t}-\tau, \bar{t}]$ we have $0 \leq x(t) \leq \bar{x}$ and therefore, $x(t) \leq x_{m}$. This shows that $x_{m}$ is the maximal possible value for the positive solution to Eq. (13). Using the same argument we see that

$$
x(t) \geq \bar{x}+\int_{t_{0}-\tau}^{t-\tau} f\left(\bar{x}+\tau f_{m}\right) d s
$$

which means that if

$$
\bar{x}+\tau\left((15 / a)^{\frac{3}{2}}\left(\sigma_{e}-c\right)^{\frac{5}{3}}+\frac{54}{5} \frac{\left(\sigma_{e}-c\right)^{\frac{7}{2}}}{a^{\frac{3}{2}}} \tau-\frac{a}{15}\left(\bar{x}+\frac{54}{5} \frac{\left(\sigma_{e}-c\right)^{\frac{5}{2}}}{a^{\frac{3}{2}}} \tau\right)^{\frac{5}{3}}\right)>0,
$$

then the solution is positive for every $t \geq 0$. It is obvious that for $\tau=0$ this inequality is satisfied and therefore, it is also satisfied for small $\tau$.

Lemma 2. If the initial function satisfies $x^{0}(h) \in[0, \bar{x}]$, for $h \in[-\tau, 0]$ and $\tau\left(\sigma_{e}-c\right)<1$, then the solution to Eq. (13) is positive for every $t>0$.

Proof. Let $\sigma_{e}-c=b$. Then Ineq. (14) takes the form

$$
(15 b / a)^{\frac{3}{2}}+\tau\left(\left(\frac{15}{a}\right)^{\frac{3}{2}} b^{\frac{5}{3}}+\frac{54}{5} \frac{b^{\frac{7}{2}}}{a^{\frac{3}{2}}} \tau-\frac{a}{15}\left((15 b / a)^{\frac{3}{2}}+\frac{54}{5} \frac{b^{\frac{5}{2}}}{a^{\frac{3}{2}}} \tau\right)^{\frac{5}{3}}\right)>0 .
$$

Dividing both sides of Ineq. (15) by $\left(\frac{b}{a}\right)^{\frac{3}{2}}$ one obtains

$$
15^{\frac{3}{2}}+b \tau\left(15^{\frac{3}{2}}+\frac{54}{5} b \tau-\frac{1}{15}\left(15^{\frac{3}{2}}+\frac{54}{5} b \tau\right)^{\frac{5}{3}}\right)>0
$$

which is equivalent to

$$
\frac{\left(15^{\frac{3}{2}}+\frac{54}{5} b \tau\right)^{\frac{5}{3}}}{15}-15^{\frac{3}{2}}-\frac{54}{5} b \tau<\frac{15^{\frac{3}{2}}}{b \tau}, \text { for } \tau>0 .
$$

Define $f_{1}(x)=\frac{\left(15^{\frac{3}{2}}+\frac{54}{5} x\right)^{\frac{5}{3}}}{15}-15^{\frac{3}{2}}-\frac{54}{5} x$ and $f_{2}(x)=\frac{15^{\frac{3}{2}}}{x}$. It is easy to see that $f_{1}(0)=0$ and $\lim _{x \rightarrow 0^{+}} f_{2}(x)=+\infty$. The function $f_{2}$ is decreasing for $x>0$ and $f_{1}(x)$ is increasing for $x>0$. Therefore, there exists a threshold value $\bar{x}$ such that $f_{1}(x)<f_{2}(x)$ for $x<\bar{x}$ and $f_{1}(x)>f_{2}(x)$ for $x>\bar{x}$. It is easy to calculate that $\bar{x}>1$. Hence, Ineq. (16) is satisfied for every $0<x<1$. This completes the proof.

The result stated in Lemma 2 is similar to that obtained in [3]. The external source of nutrient cannot be very rich to keep the solution positive. 
It is obvious that there exist the same stationary solutions to Eq. (13) as in the case of Eq. (10). Studying stability of these solutions we obtain the following result

LEMma 3. The trivial stationary solution to Eq. (13) is unstable independently of the delay. The nontrivial stationary solution $\bar{x}$ is stable for $\tau<\frac{3 \pi}{4\left(\sigma_{e}-c\right)}$ and unstable otherwise. The Hopf bifurcation occurs at $\tau=\frac{3 \pi}{4\left(\sigma_{e}-c\right)}$.

Proof. We use the method of linearization (see e.g. [11]) and obtain the characteristic equation of the form

$$
\lambda+B e^{-\lambda \tau}=0
$$

where $B=c-\sigma_{e}$ for the trivial solution and $B=\frac{2}{3}\left(\sigma_{e}-c\right)$ for the nontrivial one. We see that $B<0$ in the case of trivial solution and therefore, this solution is unstable (see [8] Corollary 5). In the second case we have $B>0$ which implies that there exists a threshold value of delay $\tau=\frac{\pi}{2 B}$. This completes the proof.

4. Numerical simulations. In this section we present the results of computer simulations. Both cases studied in the paper are considered: the model with delay in underlying apoptosis as well as the model with equal delays in both processes (underlying apoptosis and proliferation).

In all presented simulations we use constant initial function $x^{0}(t)=0.15$ for $t \in[-\tau, 0]$ and $a=30, c=4, \sigma_{e}=12$. Our aim is to show the role of the magnitude of the delay in the qualitative behaviour of tumour (temporary changes of its radius).

The need for consideration of different values of the delay follows from the biological observations showing that for different organisms, tissues and types of tumour the speed of the processes is different.

In Figs. 1, 2 and 3 changes of the behaviour of solutions to Eq. (10) with changing delay are presented (along the horizontal axes the time is given versus the values of the solutions along the vertical axes). For the chosen values of parameters we have $4 c>\sigma_{e}$ and by Lemma 1 there exists a threshold value $\tau_{c}$ of the delay (in this case $\tau_{c} \approx 0.51$

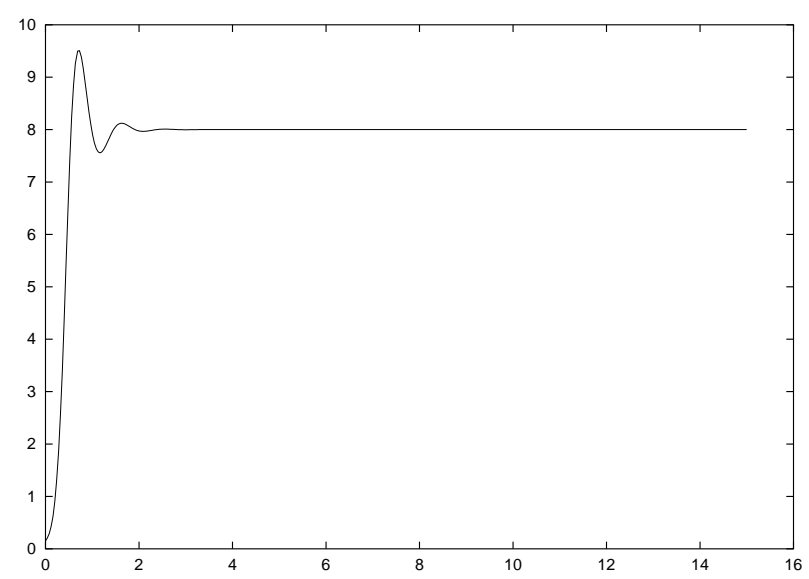

Fig. 1. An example of solution to Eq. (10) for $\tau=0.20$ 


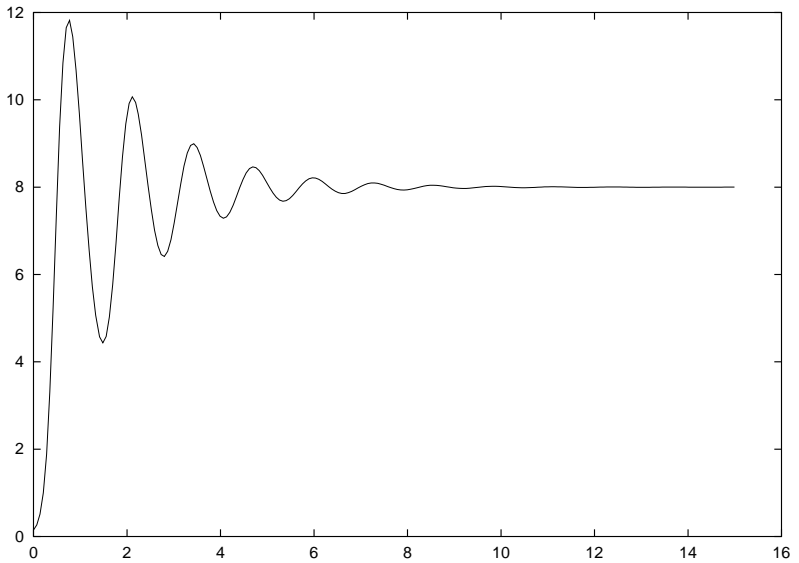

Fig. 2. An example of solution to Eq. (10) for $\tau=0.35$

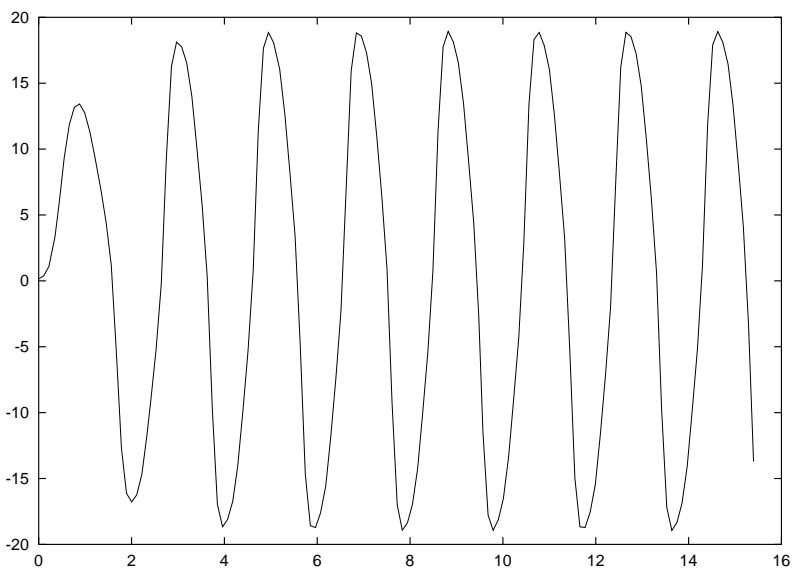

Fig. 3. An example of solution to Eq. (10) for $\tau=0.55$

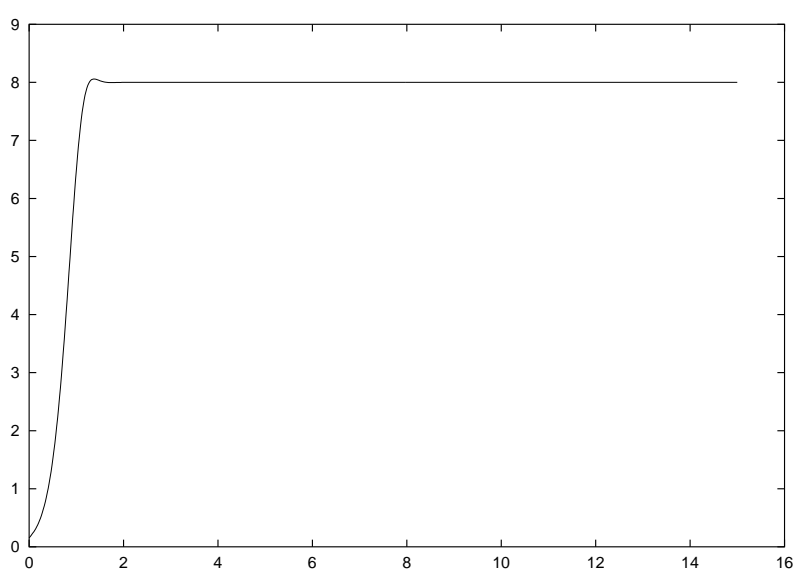

Fig. 4. An example of solution to Eq. (13) for $\tau=0.10$ 


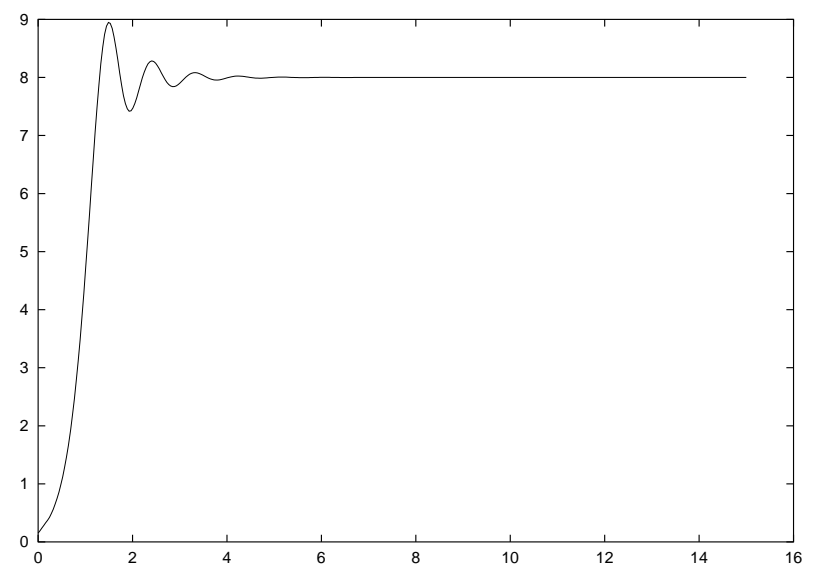

Fig. 5. An example of solution to Eq. (13) for $\tau=0.20$

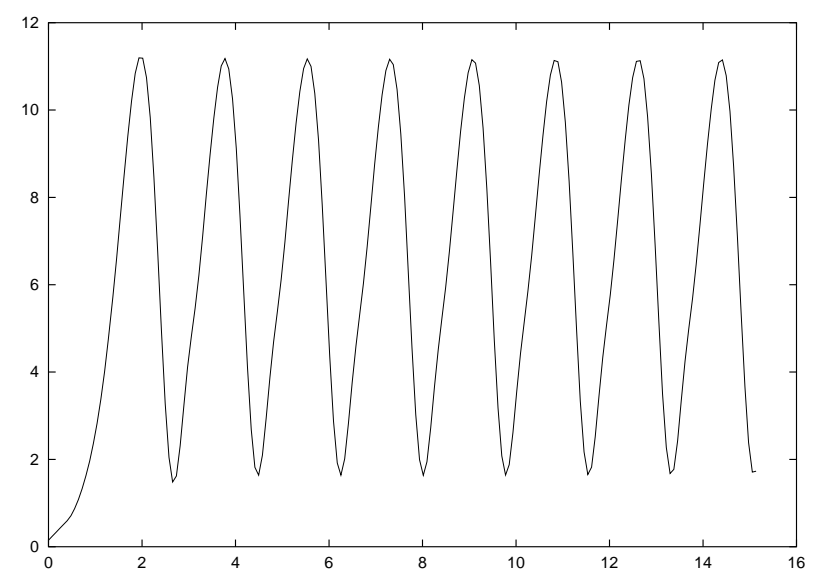

Fig. 6. An example of solution to Eq. (13) for $\tau=0.40$

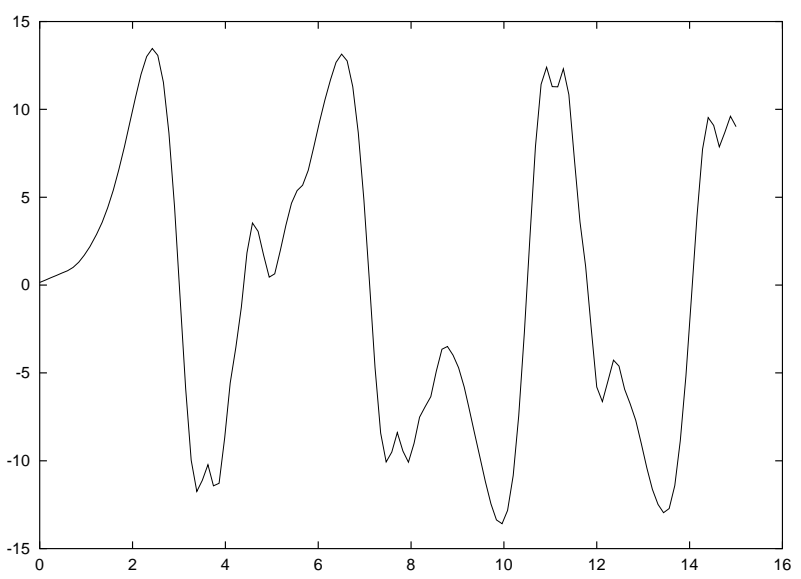

Fig. 7. An example of solution to Eq. (13) for $\tau=0.60$ 
and for this value of the delay the Hopf bifurcation occurs). For small delays the solution tends to the stationary state $\bar{x}=8$ (see Fig. 1). As delay increases damping oscillations arise (see Fig. 2). For values of the delay $\tau>\tau_{c}$ undamping oscillations are observed (see Fig. 3). For greater delays the amplitude of oscillations grows.

In Figs. 4, 5, 6 and 7 changes of the behaviour of solutions to Eq. (13) are presented. Let us note that while for some values of parameters the nontrivial solution $\bar{x}$ to Eq. (10) is stable independently of the delay, in the case of Eq. (13) the nontrivial stationary solution $\bar{x}$ is stable only when $\tau<\tau_{c}$ for every choice of the other parameter values (compare Lemma 1 and Lemma 3). For the chosen values $\tau_{c} \approx 0.295$ and for $\tau=\tau_{c}$ the Hopf bifurcation occurs. The stability of the nontrivial stationary solution $\bar{x}=8$ is observed in examples presented in Figs. 4 and 5. In Figs. 6 and 7 undamping oscillations are observed (there $\tau>\tau_{c}$ ). As can be seen, in some cases with undamping oscillations the solution is nonnegative (see Fig. 6) while in some cases it becomes negative (see Fig. $7)$. In practice if there exists $\bar{t}$ such that $x(\bar{t})=0$ and $x(t)<0$ for $t>\bar{t}$, then the model is not valid for $t>\bar{t}$ and it could be assumed that $x(t)=0$ for $t \geq \bar{t}$.

The mechanism of undamping oscillations may indirectly help to treat the tumour (see [5] for details). The occurrence of positive undamping oscillations is also observed in [5], while the simulations in [4] show in every case with undamping oscillations that the solution becomes negative. We could make a conclusion that our model with delays in both processes is more realistic in this sense in comparison with the model presented in $[4]$.

Both of our models are valid under the assumptions $R(t)<R_{c}$ (see Eq. (7)) and therefore, $\left(\frac{15\left(\sigma_{e}-c\right)}{a}\right)^{\frac{3}{2}}<R_{c}$, which is equivalent to $\left(15\left(\sigma_{e}-c\right)\right)^{3}<6 \sigma_{e} a^{2}$.

5. Discussion. In the paper we have studied the behaviour of solutions to the model of uniformly proliferating tumour with delays in underlying apoptosis only and in both cellular processes - proliferation and apoptosis. In [3, 4] similar analysis was done for the cases with delay in proliferation process only and in regulatory apoptosis. The models studied in $[3,4]$ have the following form:

$$
\dot{x}(t)=-c x(t)+\sigma_{e} x(t-\tau)-\frac{a}{15} x^{\frac{5}{3}}(t-\tau),
$$

for the case with delay in proliferation process;

$$
\dot{x}(t)=\left(\sigma_{e}-c\right) x(t)-\frac{a}{15} x^{\frac{5}{3}}-\theta\left(\sigma_{e}-\sigma_{h}\right) x(t-\tau)+\theta \frac{a}{15} x^{\frac{5}{3}}(t-\tau),
$$

for the case with delay in regulatory apoptosis.

It is easy to see that Eqs. (10), (13) and (17) differ from each other only in the place where the delay occurs. In Eq. (18) there are two additional terms which describe the process of regulatory apoptosis $\left(\theta\right.$ reflects the importance of regulatory apoptosis and $\sigma_{h}$ measures the optimal proliferation rate of MCS).

In all papers (i.e. $[3,4]$ and this one) nonnegativity of solutions to the models was investigated. The asymptotic behaviour of solutions was analysed and the possibility of the Hopf bifurcation was checked. The only common property of all studied equations is instability of the trivial stationary solution. It occurs that Eqs. (13) and (17) have similar properties. Namely, for both equations we have 
1. there are some conditions guaranteeing nonnegativity of solutions for nonnegative initial function and these conditions are connected with the difference between coefficients $\sigma_{e}$ and $c$ (the external source of nutrient cannot be rich to keep the solution under control);

2. the nontrivial stationary solution is unstable independently of the delay;

3. the nontrivial stationary solution $\bar{x}$ (the same stationary solution for Eqs. (10), (13) and (17)) is stable for small delays;

4. the Hopf bifurcation is possible for some parameter values.

It is not surprising that Eq. (18) differs from the other ones due to its different form. The most important difference is connected with the existence of nontrivial stationary solutions. Whereas for Eqs. (10), (13) and (17) the solution $\bar{x}>0$ always exists and it is the same for these models, the existence of nontrivial stationary solution to Eq. (18) depends on the magnitude of $\theta$. Eqs. (10) and (18) have another common property - there are no clear properties guaranteeing nonnegativity of solutions.

All the presented properties show that the behaviour of solutions strongly depends on the place where the delay occurs. Similar analysis with similar conclusions was presented e.g. in [12] for the logistic equation with delay. At this stage of knowledge we can say that Eqs. (13) and (17) are better than the other two. We suppose that analysis of the model with different delays for proliferation and apoptosis will show us the best way.

\section{References}

[1] J. Adam and S. A. Maggelakis, Diffusion regulated growth characteristics of a spherical prevascular carcinoma, Bull. Math. Biology 52 (1990), 549-589.

[2] M. Bodnar, The nonnegativity of solutions of delay differential equations, Appl. Math. Letters 13 (2000), 91-95.

[3] M. Bodnar and U. Foryś, Time delays in proliferation process for solid avascular tumour, Math. Comp. Modelling 37 (2003), 1201-1209.

[4] M. Bodnar and U. Foryś, Time delays in regulatory apoptosis for solid avascular tumour, Math. Comp. Modelling 37 (2003), 1211-1220.

[5] H. M. Byrne, The effect of time delays on the dynamics of avascular tumour growth, Math. Biosciences 144 (1997), 83-117.

[6] H. M. Byrne and M. A. J. Chaplain, Growth of non-necrotic tumours in the presence and absence of inhibitors, Math. Biosciences 130 (1995), 151-181.

[7] H. M. Byrne and M. A. J. Chaplain, Growth of necrotic tumours in the presence and absence of inhibitors, Math. Biosciences 135 (1996), 187-216.

[8] U. Foryś, On the Mikhailov criterion and stability of delay differential equations, preprint Warsaw University RW 01-14 (97), (2001).

[9] U. Foryś, Biological delay systems and the Mikhailov criterion of stability, J. Biol. Sys. 12 (2004), 1-16.

[10] H. P. Greenspan, Models for the growth of a solid tumour by diffusion, Stud. Appl. Math. 52 (1972), 317-340.

[11] J. Hale, Theory of Functional Differential Equations, Springer Verlag, New York, 1977.

[12] R. Kowalczyk and U. Foryś, Qualitative analysis on the initial value problem to the logistic equation with delay, Math. Comp. Modelling 35 (2002), 1-13.

[13] P. W. Reddlen, S. Cameron and H. R. Horvitz, Phagocytosis promotes programmed cell death in C. elegans, Nature 412 (2001), 198-202. 